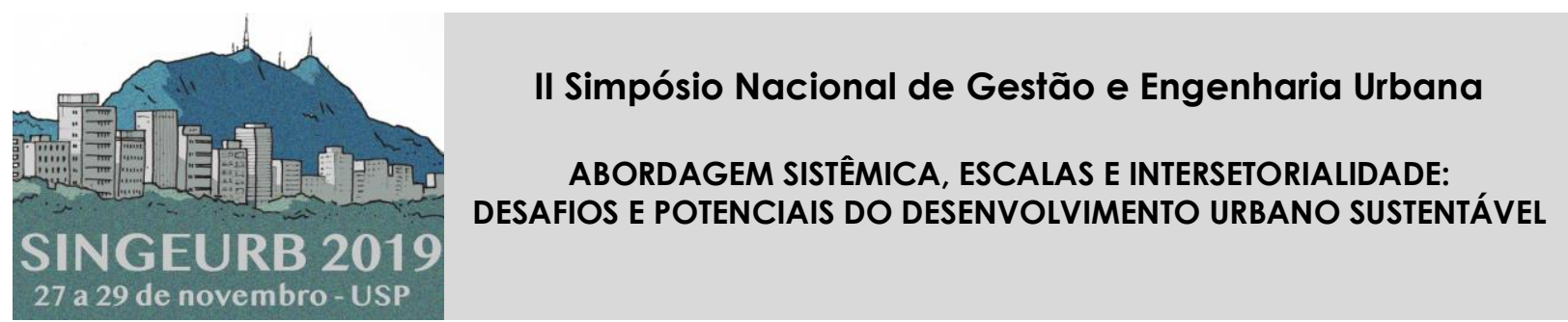

\title{
Habitação apropriada para pessoa idosa: aspectos de saúde e bem-estar ${ }^{1}$
}

\section{Housing appropriate for elderly person: aspects of health and well- being}

\author{
Campos, Marília'; Günther, Wanda Maria Risso2; \\ 1 Faculdade de Saúde Pública da Universidade de São Paulo. Rua São \\ Sebastião, n. 80, apto 64, Saúde, São Paulo, Brasil. mariliafcampos@usp.br \\ 2 Faculdade de Saúde Pública da Universidade de São Paulo. \\ wgunther@usp.br
}

\begin{abstract}
RESUMO
Neste trabalho será abordada a tendência ao aumento do número de pessoas idosas no Brasil e a urgência do fomento de políticas públicas habitacionais específicas para pessoas dessa faixa etária. Ressalta-se a necessidade de provisão de habitação apropriada para pessoas idosas, de modo a atender às necessidades quanto à acessibilidade, segurança e conforto, dadas as fragilidades inerentes a essa fase da vida. Tem por objetivos: apresentar o programa de Habitação de Interesse Social Vila dos Idosos, viabilizado pela Prefeitura do Município de São Paulo e COHAB, explanar sobre a relação entre condições de moradia e bem-estar e propor diretrizes quanto à segurança, acessibilidade e conforto para habitação apropriada a idosos. O método contou com revisão bibliográfica e documental e inspeção de campo na área de estudo. Este trabalho integra pesquisa de mestrado, no Programa Ambiente, Saúde e Sustentabilidade e relaciona-se ao Subtema 3 na medida em que se refere à inclusão da população geriátrica vulnerável nas políticas públicas de planejamento urbano. Como resultados parciais apontam-se que: (i) o Programa de Locação Social é viável para a provisão de habitação de interesse social, (ii) adequações na moradia são necessárias e reduzem o risco de queda, proporcionando autonomia e melhoria do bem-estar.
\end{abstract}

Palavras-chave: Habitação, Políticas públicas, Saúde da pessoa idosa.

\begin{abstract}
This paper will discuss about the tendency to increase the number of elderly people in Brazil and the urgency of promoting specific public housing policies for people of this age group. It is necessary to provide adequate housing for the elderly, in order to meet the needs for accessibility, safety and comfort, because of the fragilities inherent to this phase of life. Its objectives are: to present the Social Interest Housing Program for the Elderly, made possible by the Municipality of São Paulo and COHAB, to explain the relationship between living conditions and well-being, and to propose guidelines regarding safety, accessibility and comfort for
\end{abstract}

\footnotetext{
${ }^{1}$ CAMPOS, Marília; GÜNTHER, Wanda Maria Risso. Habitação apropriada para pessoa idosa: aspectos de saúde e bem-estar. In: II SIMPÓSIO NACIONAL DE GESTÃO E ENGENHARIA URBANA: SINGEURB, 2019, São Paulo. Anais... Porto Alegre: ANTAC, 2019.
} 
adequate housing for the elderly. The method was based on bibliographical and documentary review and field inspection in the study area. This work integrates master's research in the Environment, Health and Sustainability Program and relates to Subtheme 3 insofar as it refers to the inclusion of the vulnerable geriatric population in public urban planning policies. As partial results, it is pointed out that: (i) the Social Lease Program is feasible for the provision of social housing, (ii) adequate housing is necessary and reduces the risk of falling, providing autonomy and improved well-being.

Keywords: Housing, Public policy, Health of the elderly.

\section{INTRODUÇÃO}

O aumento da expectativa de vida, assim como as mudanças que vêm ocorrendo na estrutura familiar da sociedade contemporânea, é fator que aumenta o número de pessoas idosas vivendo sozinhas. As redes familiares são atualmente menores, mais complexas e geograficamente mais dispersas, o que dificulta a provisão de cuidados para com familiares idosos.

Dadas as dificuldades inerentes à faixa etária, muitas pessoas idosas acabam recorrendo à moradia que ofereça assistência necessária, seja por não receberem atenção de familiares, dependerem de cuidados especiais ou falta de condições para financiar um imóvel. Assim, considerando a tendência da população brasileira ao envelhecimento, torna-se necessário incluir esse grupo nas políticas públicas voltadas para habitação de interesse social, reforçando-se o oferecimento de serviços de assistência social.

Nesse contexto, implementar políticas públicas habitacionais direcionadas a pessoas idosas implica em considerar aspectos como segurança, acessibilidade e conforto para proporcionar bem-estar, em termos de condições ambientais, moradia e aspectos que incrementem a saúde e qualidade de vida desse grupo etário.

\section{PROBLEMATIZAÇÃO}

A tendência de aumento da população idosa implicará na elevação do déficit de políticas públicas habitacionais voltadas para esta faixa etária, em especial à população de baixa renda (MONTEIRO, 2012). O censo de 2010 apontou que a população com mais de 65 anos (7,4\% do total) aumentou mais de 50\% desde o censo de 1991 (4,8\%) (IBGE, 2010).

O envelhecimento deve ser analisado com cuidado, pois trata-se de refletir sobre a sociedade na qual se está inserido, percebendo-se que existem inúmeras velhices (COUTRIM, 2006). Estudar a complexidade do envelhecimento torna-se urgente em países com rápido incremento da população idosa, com vistas a desenvolver estratégias para garantir que as necessidades dessa população sejam supridas com qualidade (de DEUS, 2010).

A autonomia do idoso é importante aspecto no processo de envelhecimento. Villafuert et al. (2011) destacam a importância de políticas públicas considerarem os pilares estabelecidos pela Organização Mundial da Saúde (OMS) para envelhecimento ativo: saúde, participação e segurança, como prevenção aos riscos de adoecimento, dada a fragilidade pertinente à idade avançada. Porém, no Brasil, são visíveis entraves como falta de recursos financeiros, gestão ineficiente, falta de interesse do poder público, entre outros problemas de ordem político-econômica (MONTEIRO, 2012).

Nesse sentido, este trabalho trata de caso específico de programa de habitação de interesse social, com foco nas condições de moradia e bem-estar, visando identificar pontos importantes a serem considerados nas habitações apropriadas e sustentáveis para idosos. 


\section{OBJETIVOS}

Apresentar o programa de Habitação de Interesse Social Vila dos Idosos, explanar sobre a relação entre condições de moradia e bem-estar e propor diretrizes de segurança, acessibilidade e conforto em habitação apropriada para pessoas idosas.

\section{METODOLOGIA}

O método envolveu revisão bibliográfica e documental e inspeção de campo. Foi realizada busca bibliográfica nas bases PubMed e Dedalus, com as palavras-chave habitação, pessoa idosa e saúde ambiental, (português e inglês), nos últimos 20 anos, e em livros da área. A inspeção contou com anotação em caderno e registro fotográfico. A seleção da área de estudo considerou o Conjunto Habitacional específico para idosos, viabilizado a partir de política pública municipal.

\section{ASPECTOS DA HABITAÇÃO PARA PESSOA IDOSA}

\subsection{Vila dos Idosos - São Paulo}

O Conjunto Habitacional de Interesse Social Vila dos Idosos, situado no Bairro do Pari, município de São Paulo, caracteriza-se como programa de habitação social inovador, comparando-se a programas de moradia geralmente implantados em áreas periféricas e réplicas de projetos padronizados. Segundo Anitelli e Tramontano (2017), o projeto de arquitetura considerou: i) as características do terreno disponibilizado pela PMSP; ii) moradias (145 unidades) a grupo específico, com renda máxima de três salários mínimos e idade superior a 60 anos; e iii) repasse da moradia por arrendamento.

O projeto de 2003, do escritório de arquitetura Vigliecca \& Associados viabilizado pela Prefeitura Municipal de São Paulo (PMSP) em parceria com a Companhia Metropolitana de Habitação (COHAB), insere-se em terreno irregular com $2.270 \mathrm{~m}^{2}$ (BEDOLINI, 2014). A construção do conjunto habitacional, finalizada em 2007, apresenta dois tipos de moradia: quitinete e apartamento.

Vinculado a um componente do Programa Morar no Centro, denominado Programa de Locação Social (PLS), o conjunto inova por ter as unidades habitacionais locadas por meio de arrendamento, com parte do aluguel subsidiado pela PMSP (ANITELLI e TRAMONTANO, 2017). Este aspecto viabilizou a oferta de moradia para pessoas vivendo em situação precária em áreas de risco ou locais insalubres (cortiços) para habitações adequadas ao envelhecimento saudável (de DEUS, 2010).

Embora a Vila dos Idosos ofereça infraestrutura adequada para a acomodação dos beneficiados, nota-se que algumas soluções de projeto podem não contribuir para viabilizar conforto, segurança e acessibilidade mesmo nessas habitações.

\subsection{Habitação apropriada a pessoas idosas}

Embora ampla parcela da população idosa seja considerada capaz e autônoma, muitos passam a sentir dificuldades no cotidiano com as transformações naturais do corpo na velhice. Entre elas: a deficiência motora, com o decréscimo da força nas mãos e pernas, deficiências sensoriais e cognitivas, como queda da eficiência da visão, audição e dificuldades com a fala. O declínio do desempenho psicomotor causa diminuição da capacidade de memorizar e de se orientar espacialmente, dificultando a execução de atividades cotidianas (IGIMA, 2009).

Nesse contexto, a vida da pessoa idosa geralmente torna-se centrada no ambiente doméstico, o qual passa a exigir adaptações estruturais e funcionais para viabilizar conforto e bem-estar. No Brasil, a norma técnica NBR 9050/2004 estabelece critérios e especificações técnicas para avaliação de acessibilidade em edificações, mobiliário, espaços e equipamentos urbanos. 
Destaca-se ainda a importância de elementos estruturais específicos, como a construção de bancos (utilizados até mesmo em patamares de rampas ou escadas) e projetados de acordo com normas técnicas específicas. Assim, patamares podem se caracterizar como área de descanso, valorizando a transição entre diferentes ambientes (BESTETTI, 2006). Escadas também devem ser providas de corrimãos em ambos os lados, para apoio e segurança no deslocamento vertical, em especial no sentido de descenso.

Considerando a redução de mobilidade, Valins (1988) aconselha adequações que eliminem riscos de tropeções, para evitar quedas. Torna-se necessária boa iluminação e destacar mudanças de nível ou direção com sinalização e contraste de cor e textura. Como apoio complementar, é aconselhável disponibilizar sistema de alarme, caso haja necessidade de assistência.

Ambientes representados por áreas molhadas merecem atenção, equipamentos específicos e adaptações. Para Nakano (2003) a segurança no banheiro requer instalar barras de apoio no boxe e junto ao lavatório e vaso sanitário, cujo assento recomenda-se ser elevado entre 48 e $50 \mathrm{~cm}$ do chão. Além disso, torneiras de fácil manuseio são essenciais, assim como portas com abertura para fora, que não bloqueiam a entrada em caso emergencial. Ainda é apropriado piso antiderrapante e tapete de borracha no boxe.

Instalações elétricas são relevantes nessas habitações. Aliada à necessidade da previsão de tomadas e interruptores em pontos estratégicos, de fácil acesso, sua localização deve permitir o alcance com tranquilidade, estando sentado ou em pé.

A composição do mobiliário necessita avaliação desde sua real necessidade, utilidade e disposição em termos espaciais. Nakano (2003) destaca a atenção com móveis e instalação de prateleiras em alturas variáveis, para facilitar o acesso. A distribuição do mobiliário deve permitir flexibilidade no uso e viabilizar a circulação em qualquer condição, inclusive com mobilidade reduzida, em andador ou cadeira de rodas (BESTETTI, 2006).

Por outro lado, recomendações indicadas para ambientes individuais são também aplicáveis a espaços de convivência. Áreas externas necessitam ter elementos estruturais incorporados no projeto, para viabilizar a prática de exercícios físicos, que contribuem para o equilíbrio e coordenação motora e atenuam a perda de massa muscular e os riscos de osteoporose, doenças cardíacas e pulmonares (IGIMA, 2009).

Apesar do foco da concepção e projeto da Vila dos Idosos ser a pessoa idosa, verificam-se aspectos que representam risco à segurança e que não contribuem para o bem-estar dos moradores:

- O conjunto oferece diferentes espaços para sociabilidade (salão de jogos, espaços livres, horta e academia ao ar livre), porém, nota-se falta de planejamento cuidadoso desses espaços, como caminhos delimitados com piso apropriado ou aplicação de diferentes cores nos ambientes de integração;

- Como soluções plausíveis do condomínio citam-se: melhorar o sistema de iluminação, destacar o piso e o espelho dos degraus das escadas com cores diferentes, melhorar a sinalização e instalar corrimão em todas as áreas de circulação externa;

- Apesar do edifício oferecer elevadores, verificou-se acessibilidade para pessoas com mobilidade reduzida prejudicada no interior das quitinetes e apartamentos;

- A mobília dos ambientes internos, não planejada antecipadamente e, em geral, a cargo do morador, podem não proporcionar maior flexibilidade e acessibilidade, como quando previstas com móveis planejados.

Ige et al. (2018) relatam resultados de estudos sobre impactos das condições da residência na saúde dos moradores. Avaliados os resultados associados à implantação de rampas e adaptações na iluminação para proporcionar independência à pessoa idosa, houve correlação positiva entre prevenção a quedas e melhorias na saúde. Os autores comentam ainda que a interpretação da moradia como local inseguro pode acarretar efeitos negativos na saúde mental dos moradores. 
Segundo Ige et al. (2018), estudos de Leventhal e Brooks-Gunn (2003 e 2004), com intuito de verificar efeitos do entorno da residência na saúde dos moradores, avaliaram benefícios da realocação de pessoas de alojamento público em região carente para moradia própria em áreas adequadas. Os resultados apontaram redução nos sintomas de depressão em adultos, - que fortalece a associação entre condições de moradia e consequências na saúde humana, assim como confirma a importância da provisão de habitação de boa qualidade a preços acessíveis a grupos vulneráveis.

\section{CONCLUSÕES}

A alta demanda por moradia no Brasil vem sendo parcialmente atendida por políticas públicas habitacionais que envolvem financiamento para provisão de Habitação de Interesse Social. Porém, muitas vezes, pessoas idosas acabam excluídas por serem abandonadas pelos parentes ou por falta de condições de financiar um imóvel, seja por renda insuficiente ou fragilidades físicas e mentais.

O Programa de Locação Social caracteriza-se como alternativa viável para a provisão de habitação para pessoas idosas, pois requer baixa porcentagem da renda do beneficiado e garante a zeladoria do local e assistência por parte do poder público. A Vila dos Idosos é exemplo relevante de inclusão social de grupo vulnerável, quando saúde e qualidade de vida requer maior atenção. Portanto, configura-se em proposta a ser difundida e implementada em âmbito nacional, considerando o incremento do envelhecimento da população e demanda crescente por moradia para este público.

No entanto, é relevante considerar aspectos de segurança e bem-estar, vinculados diretamente à saúde. Tais aspectos envolvem elementos de projeto e construtivos, seleção e distribuição do mobiliário e adaptações necessárias à faixa etária, assim como planejamento cuidadoso do uso dos ambientes e elementos presentes. Verificou-se que, mesmo em empreendimento voltado a acomodar os beneficiados em estrutura adequada, há possibilidade de aprimorar as instalações, mobiliário e equipamentos, com reflexos na promoção da saúde.

\section{REFERÊNCIAS}

ANITELLI, F.; TRAMONTANO, M. Vila dos Idosos: novos insumos para a política habitacional, novos parâmetros para a arquitetura, novas resultantes urbanas. Oculum Ensaios, Campinas, v.14, n.1, p.63-80, 2017. Disponível em:< http://periodicos.puc-

campinas.edu.br/seer/index.php/oculum/article/view/3246/2448> Acesso em 20 maio 2018.

BEDOLINI, A. C. B. A Vila dos Idosos de Héctor Vigliecca: Uma reflexão sobre o "fazer arquitetura". In: III ENCONTRO DA ASSOCIAÇÃO NACIONAL DE PESQUISA E PÓS-

GRADUAÇÃO EM ARQUITETURA E URBANISMO, 2014, São Paulo. Disponível em: <http://www.anparq.org.br/dvd-enanparq-3/htm/Artigos/SC/ORAL/SC-HDC022_BEDOLINI.pdf > Acesso em 01 maio 2018.

BESTETTI, M. L. T. Habitação para Idosos. O trabalho do arquiteto, arquitetura e cidade. 2006. Tese (Doutorado em Arquitetura e Urbanismo) - Faculdade de Arquitetura e Urbanismo, Universidade de São Paulo, São Paulo, 2006.

COUTRIM, R. M. da E. Algumas considerações teóricas e metodológicas sobre estudos de sociologia do envelhecimento. Revista Brasileira de Geriatria e Gerontologia, Rio de Janeiro, v. 9, n. 3, 2006. Disponível em $<$ http://revista.unati.uerj.br/scielo.php?script=sci_arttext\&pid=\$180998232006000300006\&lng=pt\&nrm=iso>. Acesso em 04 fev. 2019.

De DEUS, S. I. A. Um modelo de moradia para idosos: o caso da Vila dos Idosos do Pari - São Paulo (SP). Caderno Temático Kairós Gerontologia, São Paulo, v. 13 , n. 8, p. 195-213, 2010. 
Disponível em: <https://revistas.pucsp.br/kairos/article/view/6922/5014> Acesso em 10 jul. 2019.

IGE, J.; PILKINGTON, P.; ORME J.; WILLIAMS, B.; PRESTWOOD, E.; BLACK, D.; CARMICHAEL, L.; SCALLY, G. The relationship between buildings and health: a systematic review. Journal of Public Health, Oxford, 2018. Disponível em:

$<$ https://academic.oup.com/jpubhealth/advance-

article/doi/10.1093/pubmed/fdy138/5076115> Acesso em 01 set. 2018.

IGIMA, J. C. Centro de Convivência Saúde e Bem-Estar para idosos na cidade de São Paulo. Trabalho Final de Graduação. Faculdade de Arquitetura e Urbanismo - Universidade de São Paulo, 2009.

IBGE - INSTITUTO BRASILEIRO DE GEOGRAFIA E ESTATíSTICA. Censo Demográfico 2010.

Disponível em:

<https://ww2.ibge.gov.br/home/estatistica/populacao/censo2010/default.shtm> Acesso em 10 jul. 2019.

MONTEIRO, L. C. A. Políticas Públicas Habitacionais para idosos: um estudo sobre os

condomínios exclusivos. 2012. Dissertação (Mestrado em Engenharia Urbana) - Centro de Ciências Exatas e de tecnologia, Universidade Federal de São Carlos, São Carlos, 2012.

NAKANO, F. Mobiliário para idosos: Acessibilidade para todos. Trabalho Final de Graduação. Faculdade de Arquitetura e Urbanismo - Universidade de São Paulo, 2003.

VALINS, M. Housing for elderly people. A Guide for Architects, Interior Designers and their

Clients. London: The Architectural Press, 1988.

VILLAFUERTE, B. E. P.; SILLER, S. T.; VAZQUEZ, T. G.; SERRANO, M. M.; Apoio social y condiciones de vida de adultos mayores que viven en la pobreza urbana en Mexico. Scielo, Cad. Saúde Pública, Rio de Janeiro, v. 27, n.3, p. 460-470, 2011 . Disponível em:

< https://scielosp.org/pdf/csp/2011.v27n3/460-470/es> Acesso em 22 maio 2018. 